\title{
Management of Severe ARDS: New Strategies and Ongoing Challenges
}

In this issue of ReSPIRATORY CARE, articles by Spina et $\mathrm{al}^{1}$ and Gallo de Moraes et $\mathrm{al}^{2}$ focus on the institutional development of an early and dedicated approach to manage subjects with severe ARDS. Although the techniques used are different and the studies underpowered for clinical outcomes, the results converge to the same conclusion: specialized and dedicated teams and protocols facilitate implementing and customizing advanced treatment and rescue strategies in patients with severe ARDS.

Guidelines and recommendations on mechanical ventilation have flourished in the last decade, especially for the management of ARDS. A better understanding of lung injury, its effect on lung mechanics, and their interaction with mechanical ventilation has led to increasingly complex considerations regarding the ventilatory management of these patients. For instance, whereas ventilator-induced lung injury is a well-known concept, ${ }^{3}$ other concepts have been recently added to the lexicon (eg, patient self-inflicted lung injury, myotrauma, diaphragm-protective ventilation) ${ }^{4-6}$ Key studies in the last 20 years have built a basic framework of treatment for ARDS: pressure- and volumelimited ventilation, ${ }^{7,8}$ higher PEEP, ${ }^{9}$ and prone positioning $^{10}$ for the most severe cases of ARDS. However, some patients still fail initial management. Moreover, broad-scale observational data such as the LUNG SAFE study ${ }^{11}$ clearly indicate that there is room for improvement, even in the early identification of ARDS and implementing evidencebased interventions.

Following the implementation of care based on published guidelines, ${ }^{12}$ how and when should a patient with severe ARDS be referred for more specialized care? What is the optimal time period after which you can say that your patient has failed an intervention (eg, prone positioning)? When should clinicians refer patients for venovenous extracorporeal membrane oxygenation (VV-ECMO)? For those patients supported with VV-ECMO, what is the best

Dr Fan has disclosed relationships with ALung Technologies, MC3 Cardiopulmonary, and Fresenius. Dr Ratano has disclosed no conflicts to of interest.

Correspondence: Eddy Fan MD PhD, Toronto General Hospital, 585 University Avenue, PMB 11-123, Toronto, Ontario, Canada, M5G 2N2. E-mail: eddy.fan@uhn.ca.

DOI: $10.4187 /$ respcare. 07763 ventilation modality during ECMO? Both Spina et $\mathrm{al}^{1}$ and Gallo de Moraes et $\mathrm{al}^{2}$ make suggestions for some of these questions, but perhaps the most important message is that a “one size fits all” strategy doesn't exist.

See the Related Article on Page 413

The first question that could be asked is how do you define failure of your primary strategy? Moreover, how far does your primary strategy go? When do you need a rescue team? Whereas implementing lung protective ventilation and neuromuscular blockade are relatively straightforward in most patients and centers, barriers to prone positioning continue to exist. ${ }^{11,13}$ Although supported by clinical trial data, only $16 \%$ of subjects with severe ARDS were proned in the LUNG SAFE study, ${ }^{11}$ and only $50-60 \%$ of subjects in the EOLIA trial ${ }^{14}$ were proned before randomization to ECMO. Furthermore, how do you define proning failure? The EOLIA trial ${ }^{14}$ had clear criteria for ECMO referral and proning became mandatory after a change in the protocol, but failure of proning was not well defined because no specific definition exists. This lack of clarity on thresholds for success or failure creates issues with referring patients too early or too late to ECMO centers.

How best to move forward? Possibilities include more research, standardized treatment, and building a network of care (eg, the hub and spoke model), all of which are not necessarily mutually exclusive. Standardization is the holy grail of modern medicine because this approach is rational and evidence-based, as well as more cost-effective. Though we do not argue against standardization for the initial management of ARDS, limitations still exist. Standards of care are well defined, ${ }^{15}$ but their application is variable. ${ }^{11}$ There are multiple barriers to standardization, but 3 major factors can be identified: patients, physicians, and health care resources. ARDS, as defined by the Berlin criteria, ${ }^{16}$ is a heterogeneous syndrome, and each patient is a unique case. Personalized or precision medicine is expanding in multiple domains of medicine, ${ }^{17-19}$ including the identification of ARDS subphenotypes that may respond differently to treatments. ${ }^{20}$ Another theoretical example is the recent re-analysis of the ART trial, ${ }^{21}$ which suggests that recruitment maneuvers may be beneficial in some subgroups of patients 


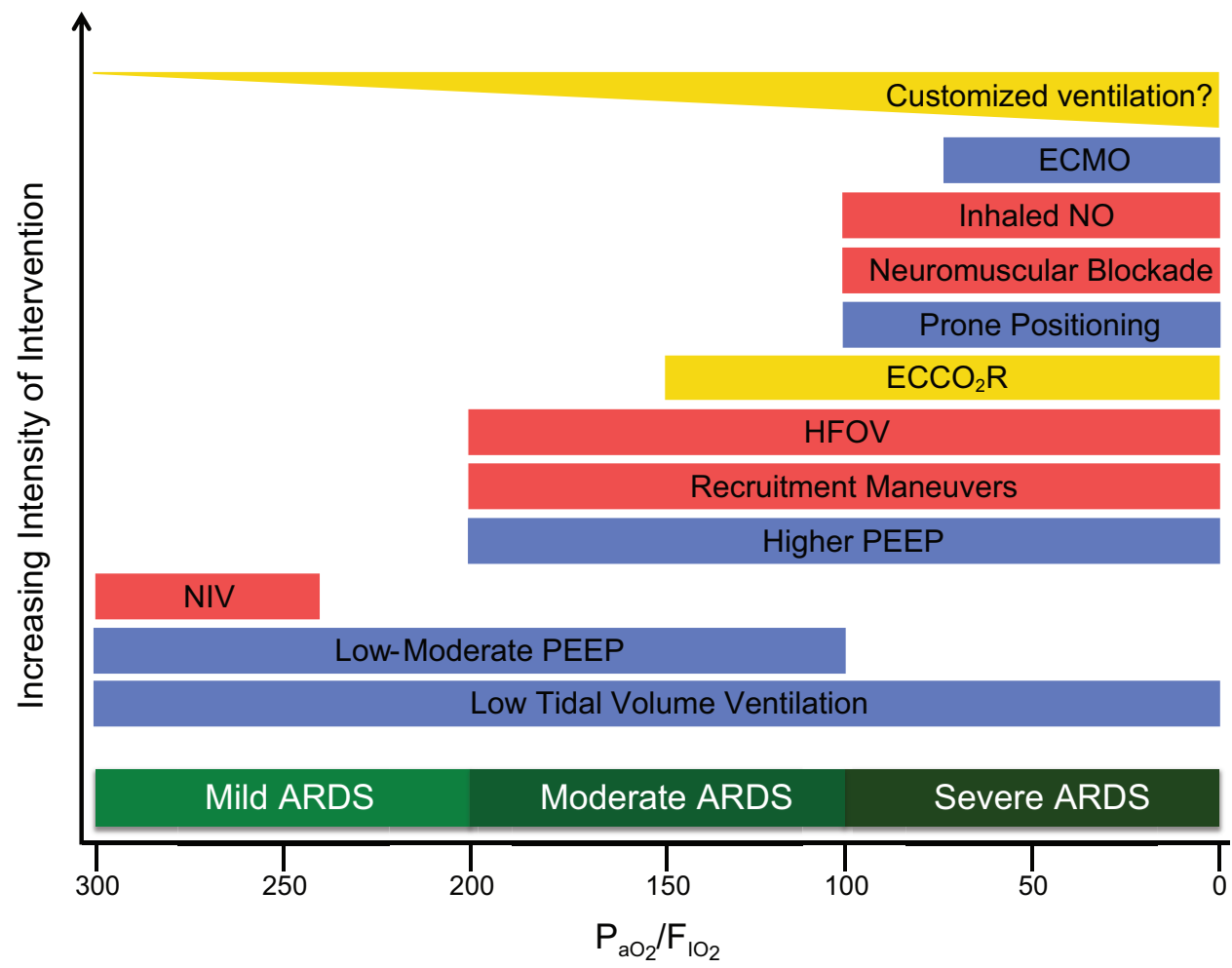

Fig. 1. An approach to interventions for the management of ARDS. The figure depicts potential interventions that could be used in the management of patients with ARDS. Boxes in blue represent interventions supported by the current evidence. Boxes in red represent interventions that are not recommended for routine use according to the current evidence. Boxes in yellow represent interventions that still require confirmation through clinical trials. Customized mechanical ventilation may include the deployment of institution-specific protocols or teams, along with techniques such as esophageal manometry and electrical impedance tomography. Over time, and with more data, various aspects of this approach may change; some interventions may be found not to be useful, while others may be added, and thresholds for use may change. ECMO = extracorporeal membrane oxygenation; $\mathrm{ECCO}_{2} \mathrm{R}=$ extracorporeal $\mathrm{CO}_{2}$ removal; $\mathrm{HFOV}=$ high-frequency oscillatory ventilation; NIV = noninvasive ventilation.

identified with cluster analysis. ${ }^{22}$ Strong physiological rationale supports the use of esophageal manometry or electrical impedance tomography, which can help individualize mechanical ventilation, although clinical trials evaluating strategies based on their use are needed. We hypothesize that clinicians would begin by implementing standardized care, followed by the use of specialized institutional teams or protocols to customize mechanical ventilation from that starting point in difficult-to-manage (eg, morbid obesity) or severe cases of ARDS (Figure 1).

Observational data show us how difficult it can be even to diagnose ARDS. ${ }^{11}$ Unfortunately, simple web-based educational interventions may not have much impact on reducing under-recognition. ${ }^{23}$ However, with the increasing use of electronic medical records, automated alerts to clinicians regarding the diagnosis of ARDS may improve recognition. Once ARDS is clearly established, the next steps should be focused on optimizing mechanical ventilation. A simple checklist to verify that all evidence-based interventions (eg, low tidal-volume, low plateau pressure, neuromuscular blockade, PEEP) are applied could be used. Again, clinical decision-support systems, which have been used successfully in the ICU, ${ }^{24,25}$ could be very helpful to ensure optimal adherence. A more detailed algorithm as suggested by Gallo de Moraes et $\mathrm{al}^{2}$ can be the next step. The use of complex interventions, such as prone positioning, esophageal manometry, or electrical impedance tomography may be challenging to implement more widely. For instance, Law et $\mathrm{al}^{13}$ pointed out that smaller hospitals tend to prone less, suggesting resources and experience may be crucial factors. Conversely, the same study also reported some frequent misconceptions about prone positioning, such as the need for specific devices. This is where education of ICU teams and institutional policies could make a difference. For the moment, these interventions, along with VV-ECMO, might continue to be better performed in referral centers with higher case volumes and more experience with these techniques.

What about networks? Although large research (ARDSnet, ECMOnet, ELSO, the French REVA network) networks are well-known, they may not be representative of the daily clinical needs of most ICUs. Regional clinic-oriented networks have to be created at every level of ARDS management. As mentioned above, each facility should have a 
standardized ARDS procedure adapted to its resources. This adaptation can happen only by taking into consideration the situation of a given hospital in its region, bringing networking and referral centers to the heart of the debate. Whereas the limitations of smaller hospitals are often recognized, the limitations of referral centers should be acknowledged, too. As the number of referrals increases, referral centers may need to refuse patients due to capacity limitations. Moreover, some patients are simply too unstable to be transferred, and VV-ECMO retrieval may not be available due to transport complexities such as weather, distance, or team availability. In these situations, could smaller hospitals apply some advanced techniques of mechanical ventilation? Using remote or e-ICU systems ${ }^{26}$ as an example, a regional lung rescue team with a respiratory therapist assisted by a specialized physician could provide guidance remotely. This type of intervention may have a place in the complex landscape of critical care and help avoid overcrowding of referral centers. This would be realizable only if all the stakeholders of a network have an intimate knowledge of each other's capacity and with strong support of hospital administrators and dedicated, sustainable funding.

In conclusion, the studies by Spina et $\mathrm{al}^{1}$ and Gallo de Moraes et $\mathrm{al}^{2}$ support the need for specific tools and resources in the management of patients with severe ARDS. Early recognition of severe ARDS is the key to the potential for optimal treatment and identifying patients who may benefit from referral to a larger ARDS/ECMO center. Research must continue to answer ongoing questions on the early management of ARDS, such as how to clearly define thresholds that mark failure of the initial strategy and when it's best to refer. Building local and regional programs for the management of acute respiratory failure may overcome some of the barriers to applying international recommendations. Finally, although applying evidence-based interventions in all patients with ARDS is the main challenge, defining new management strategies could be an important step to help rationalize the use of limited resources and improve the outcome of patients with ARDS.

Damian Ratano Interdepartmental Division of Critical Care Medicine University of Toronto Toronto, Canada

Department of Medicine University Health Network and Sinai Health System Toronto, Canada Intensive Care and Burn Unit Lausanne University Hospital Lausanne, Switzerland

Eddy Fan
Interdepartmental Division of Critical Care Medicine
University of Toronto
Toronto, Canada
Department of Medicine
University Health Network and Sinai Health System
Toronto, Canada

\section{REFERENCES}

1. Spina S, Capriles M, De Santis Santiago R, Florio G, Teggia-Droghi $\mathrm{M}$, Grassi L, et al. Development of a lung rescue team to improve care of subjects with refractory acute respiratory failure. Respir Care 2020;65(4):420-426.

2. Gallo de Moraes A, Holets SR, Tescher AN, Elmer J, Arteaga GM, Schears G, et al. The clinical effect of an early, protocolized approach to mechanical ventilation for severe and refractory hypoxemia. Respir Care 2020;65(4):413-419.

3. Slutsky AS, Ranieri VM. Ventilator-induced lung injury. N Engl J Med 2013;369(22):2126-2136.

4. Brochard L, Slutsky A, Pesenti A. Mechanical ventilation to minimize progression of lung injury in acute respiratory failure. Am J Respir Crit Care Med 2017;195(4):438-442.

5. Goligher EC, Brochard LJ, Reid WD, Fan E, Saarela O, Slutsky AS, et al. Diaphragmatic myotrauma: a mediator of prolonged ventilation and poor patient outcomes in acute respiratory failure. Lancet Respir Med 2019;7(1):90-98.

6. Schepens T, Goligher EC. Lung- and diaphragm-protective ventilation in acute respiratory distress syndrome: rationale and challenges. Anesthesiology 2019;130(4):620-633.

7. Acute Respiratory Distress Syndrome Network; Brower RG, Matthay MA, Morris A, Schoenfeld D, Thompson BT, Wheeler A. Ventilation with lower tidal volumes as compared with traditional tidal volumes for acute lung injury and the acute respiratory distress syndrome. $\mathrm{N}$ Engl J Med 2000;342(18):1301-1308.

8. Amato MBP, Meade MO, Slutsky AS, Brochard L, Costa ELV, Schoenfeld DA, et al. Driving pressure and survival in the acute respiratory distress syndrome. N Engl J Med 2015;372(8):747-755.

9. Briel M, Meade M, Mercat A, Brower RG, Talmor D, Walter SD, et al. Higher vs lower positive end-expiratory pressure in patients with acute lung injury and acute respiratory distress syndrome: systematic review and meta-analysis. JAMA 2010;303(9):865.

10. Guérin C, Reignier J, Richard J-C, Beuret P, Gacouin A, Boulain T, et al. Prone positioning in severe acute respiratory distress syndrome. N Engl J Med 2013;368(23):2159-2168.

11. Bellani G, Laffey JG, Pham T, Fan E, Brochard L, Esteban A, et al. Epidemiology, patterns of care, and mortality for patients with acute respiratory distress syndrome in intensive care units in 50 countries. JAMA 2016;315(8):788.

12. Fan E, Del Sorbo L, Goligher EC, Hodgson CL, Munshi L, Walkey AJ, et al. An official American Thoracic Society/European Society of Intensive Care Medicine/Society of Critical Care Medicine clinical practice guideline: mechanical ventilation in adult patients with acute respiratory distress syndrome. Am J Respir Crit Care Med 2017;195 (9): 1253-1263.

13. Law AC, Forbath N, O'Donoghue S, Stevens JP, Walkey AJ. Hospital-level availability of prone positioning in Massachusetts intensive care units. Am J Respir Crit Care Med. 2020 [ePub ahead of print]. doi: 10.1164/rccm.201910-2097LE

14. Combes A, Hajage D, Capellier G, Demoule A, Lavoué S, Guervilly $\mathrm{C}$, et al. Extracorporeal membrane oxygenation for severe acute respiratory distress syndrome. N Engl J Med 2018;378(21):1965-1975. 


\section{EDITORIAL}

15. Papazian L, Aubron C, Brochard L, Chiche J-D, Combes A, Dreyfuss D, et al. Formal guidelines: management of acute respiratory distress syndrome. Ann Intensive Care 2019;9(1):69.

16. The ARDS Definition Task Force. Acute respiratory distress syndrome: the Berlin definition. JAMA 2012;307(23):2526-2533.

17. Claassens DMF, Vos GJA, Bergmeijer TO, Hermanides RS, van't Hof AWJ, van der Harst P, et al. A Genotype-guided strategy for oral P2Y ${ }_{12}$ inhibitors in primary PCI. N Engl J Med 2019;381(17):16211631.

18. Helgadottir H, Rocha Trocoli Drakensjö I, Girnita A. Personalized medicine in malignant melanoma: towards patient tailored treatment. Front Oncol 2018;8:202.

19. Chung KF, Adcock IM. Precision medicine for the discovery of treatable mechanisms in severe asthma. Allergy 2019;74(9):1649-1659.

20. Calfee CS, Delucchi KL, Sinha P, Matthay MA, Hackett J, Shankar-Hari M, et al. Acute respiratory distress syndrome subphenotypes and differential response to simvastatin: secondary analysis of a randomised controlled trial. Lancet Respir Med 2018;6(9):691-698.

21. Writing Group for the Alveolar Recruitment for Acute Respiratory Distress Syndrome Trial (ART) Investigators; Cavalcanti AB, Suzumura ÉA, Laranjeira LN, Paisani DM, Damiani LP, et al. Effect of lung recruitment and titrated positive end-expiratory pressure (PEEP) vs low
PEEP on mortality in patients with acute respiratory distress syndrome: a randomized clinical trial. JAMA 2017;318(14):1335-1345.

22. Zampieri FG, Costa EL, Iwashyna TJ, Carvalho CRR, Damiani LP, Taniguchi LU, et al. Heterogeneous effects of alveolar recruitment in acute respiratory distress syndrome: a machine learning reanalysis of the Alveolar Recruitment for Acute Respiratory Distress Syndrome Trial. Br J Anaesth 2019;123(1):88-95.

23. Goddard SL, Rubenfeld GD, Manoharan V, Dev SP, Laffey J, Bellani $\mathrm{G}$, et al. The randomized educational acute respiratory distress syndrome diagnosis study: a trial to improve the radiographic diagnosis of acute respiratory distress syndrome. Criti Care Med 2018;46(5):743748.

24. East TD, Heermann LK, Bradshaw RL, Lugo A, Sailors RM, Ershler L, et al. Efficacy of computerized decision support for mechanical ventilation: results of a prospective multi-center randomized trial. Proc AMIA Symp 1999;251-255.

25. Thompson BT, Orme JF, Zheng H, Luckett PM, Truwit JD, Willson $\mathrm{DF}$, et al. Multicenter validation of a computer-based clinical decision support tool for glucose control in adult and pediatric intensive care units. J Diabetes Sci Technol 2008;2(3):357-368.

26. Celi LA, Hassan E, Marquardt C, Breslow M, Rosenfeld B. The eICU: it's not just telemedicine. Critical Care Med 2001;29(Suppl):N183N189. 